\title{
Distinguishing Zika and Dengue Viruses through Simple Clinical Assessment, Singapore
}

\author{
Gabriel Yan, ${ }^{1}$ Long Pang, ${ }^{1}$ Alex R. Cook, ${ }^{2}$ \\ Hanley J. Ho, ${ }^{2}$ Mar Soe Win, Ai Leng Khoo, \\ Joshua G.X. Wong, Chun Kiat Lee, \\ Benedict Yan, Roland Jureen, \\ Siew Seen Ho, David C. Lye, \\ Paul A. Tambyah, Yee Sin Leo, \\ Dale Fisher, Jolene Oon, \\ Natasha Bagdasarian, Angela Chow, ${ }^{3}$ \\ Nares Smitasin, ${ }^{3}$ Louis Yi Ann Chai ${ }^{3}$
}

Dengue virus and Zika virus coexist in tropical regions in Asia where healthcare resources are limited; differentiating the 2 viruses is challenging. We showed in a casecontrol discovery cohort, and replicated in a validation cohort, that the diagnostic indices of conjunctivitis, platelet count, and monocyte count reliably distinguished between these viruses.

Z ika virus and dengue virus (DENV) are arboviral infections transmitted by the Aedes mosquito. Dengue is endemic in Singapore with $>10,000$ case notifications annually (1). Although Zika virus was known through serosurveys to circulate in Southeast Asia (2), confirmed infections had been scarce until August 2016, when the first recognized outbreak in Southeast Asia occurred in Singapore, following the epidemic in the Americas (3).

Co-circulation of both viruses poses challenges to healthcare providers in distinguishing between the 2 infections. These infections have similar clinical features, including fever, rash, and myalgia. Because most patients enter the primary healthcare setting with nonspecific symptoms, we sought to determine if either infection had distinguishing symptoms, signs, or basic laboratory findings.

Author affiliations: National University Health System, Singapore (G. Yan, L. Pang, A.R. Cook, M.S. Win, S.S. Ho, P.A. Tambyah, D. Fisher, J. Oon, N. Bagdasarian, N. Smitasin, L.Y.A. Chai); National University of Singapore (L. Pang, A.R. Cook, P.A. Tambyah, D. Fisher, N. Bagdasarian, L.Y.A. Chai); Tan Tock Seng Hospital, Singapore (H.J. Ho, J.G.X. Wong, D.C. Lye, Y.S. Leo, A. Chow); National University Cancer Institute, Singapore (M.S. Win, L.Y.A. Chai); National Healthcare Group, Singapore (A.L. Khoo); National University Hospital, Singapore (C.K. Lee, B. Yan, R. Jureen)

DOI: https://doi.org/10.3201/eid2408.171883

\section{The Study}

We conducted a case-control study at the National University Hospital with ethics approval from the hospital's Institutional Review Board. Patients infected with Zika virus and DENV who were seen at the hospital in 2016 constituted the discovery cohort. We confirmed Zika virus infection through testing for viral RNA in serum or urine, as described by Lanciotti et al. (4). We confirmed DENV infection through testing for serum DENV nonstructural protein 1 (NS1) antigen (SD BIOLINE Dengue DUO Kit; Standard Diagnostics, Kyonggi-do, South Korea) or by reverse transcription PCR (5). The clinical information collected included demographics, symptomatology, examination findings, and laboratory investigations, including complete blood count (with the monocyte count automated) and liver function test.

We compared clinical characteristics of both infections by univariate logistic regression against dichotomous symptomatology and continuous laboratory parameters. We selected predictors that could differentiate Zika virus and DENV infection as input for subsequent multivariate regression models and computed the area under the receiver operating characteristic curve (AUC) to compare model performance. We validated the results in a separate cohort of Zika virus and DENV patients from Tan Tock Seng Hospital, Singapore (5). From this validation cohort, we ascertained AUC and accuracy of the derived predictors. There were no pregnant patients in either cohort. We performed all analyses with R statistical software version 3.3.1 (http:// www.R-project.org).

We identified 121 patients for the discovery study; 34 had Zika virus and 87 had DENV infection. Fifteen Zika patients $(44.1 \%)$ were male and $19(55.9 \%)$ female; 57 (65.5\%) DENV patients were male and $30(34.5 \%)$ female. Thirty-one Zika patients $(91.1 \%)$ were PCR positive by urine test and $3(8.9 \%)$ by plasma.

Zika patients sought treatment earlier in their illness than did DENV patients. Whereas viral symptoms including fever and arthralgia were common to both, differences were discernible (Figure 1). Conjunctivitis strongly indicated Zika virus infection (odds ratio [OR] 30.1, 95\% CI 9.57-94.44; $\mathrm{p}<0.001$ ). In contrast, fever (OR 0.05,

\footnotetext{
${ }^{1}$ These authors contributed equally to this article.

${ }^{2}$ These authors contributed equally to this article.

${ }^{3}$ These authors contributed equally to this article.
} 


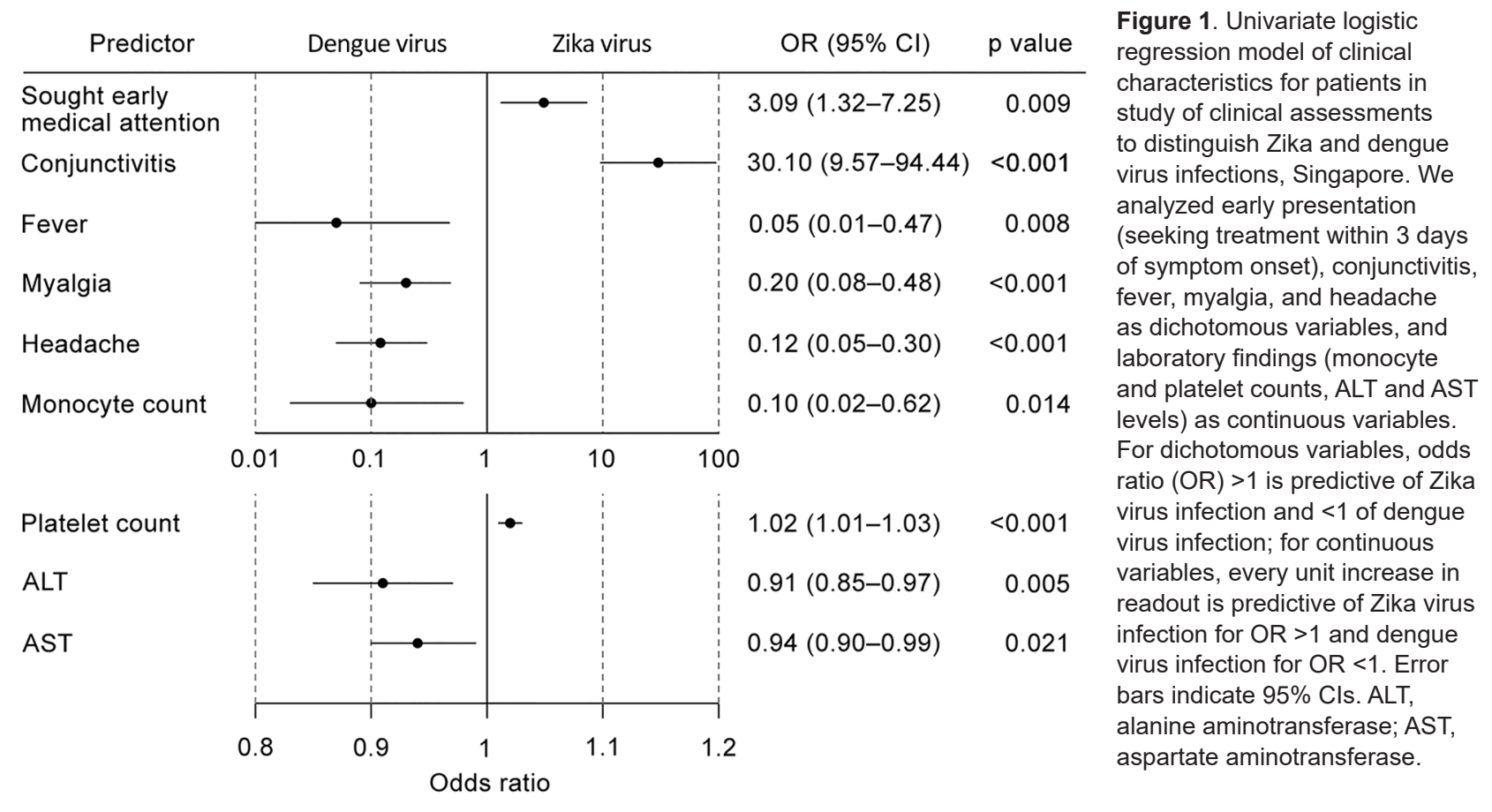

95\% CI 0.01-0.47; $\mathrm{p}=0.008$ ), myalgia (OR 0.20, 95\% CI $0.08-0.48 ; \mathrm{p}<0.001$ ), and headache (OR 0.12, 95\% CI $0.05-0.30 ; \mathrm{p}<0.001)$ were more prominent in patients with DENV infection.

Further, DENV patients tended to have thrombocytopenia (median platelet count $132 \times 10^{9} / \mu \mathrm{L}$, range $15-386 \times$ $10 \% / \mu \mathrm{L}$ ) and monocytosis (median monocyte count $0.50 \times$ $10^{9} / \mu \mathrm{L}$, range $\left.0.11-1.70 \times 10^{9} / \mu \mathrm{L}\right)$, whereas Zika patients tended to have normal platelet (median $225 \times 10^{9} / \mu \mathrm{L}$, range $\left.128-326 \times 10^{9} / \mu \mathrm{L} ; \mathrm{p}<0.001\right)$ and monocyte (median 0.35 $\times 10^{9} / \mu \mathrm{L}$, range $\left.0.13-1.00 \times 10^{9} / \mu \mathrm{L} ; \mathrm{p}=0.021\right)$ counts. The odds of Zika virus infection increased $2 \%$ with every unit $(10 \% / \mu \mathrm{L})$ increase in platelet count (OR 1.02, 95\% CI $1.01-1.03 ; \mathrm{p}<0.001$ ) (Figure 1). Lower monocyte counts were associated with Zika virus infection (OR 0.10, 95\% CI, 0.02-0.62; $\mathrm{p}=0.014)$.

Patients with DENV had biochemical evidence of liver injury with hepatic alanine aminotransferase (ALT) and aspartate aminotransferase (AST) levels $>2$ times the upper reference limit (ALT, median 51.0, range 12-465 U/L; AST, median 65, range 20-720 U/L). The reference range for ALT is 10-70 U/L, and for AST, 10-50 U/L. In contrast, Zika virus patients did not have pronounced abnormalities in albumin, ALT, AST, or alkaline phosphatase levels.

Our findings point to conjunctivitis, platelet, monocyte, ALT, and AST levels as candidate markers to differentiate Zika virus patients from DENV patients. Conjunctivitis alone had an AUC of 0.79 in identifying Zika virus patients; normal platelet count in addition to conjunctivitis increased the AUC to 0.92, and adding a normal monocyte count further improved the AUC to 0.95 (Figure 2).
The use of these 3 indices (conjunctivitis and platelet and monocyte counts) had $88 \%$ sensitivity and 93\% specificity in distinguishing Zika virus from DENV, with a diagnostic accuracy of $92 \%$. Inclusion of ALT and AST, however, did not further enhance the diagnostic capability.

We applied these 3 indices to a validation cohort consisting of $25 \mathrm{Zika}$ virus and 70 DENV patients (Table

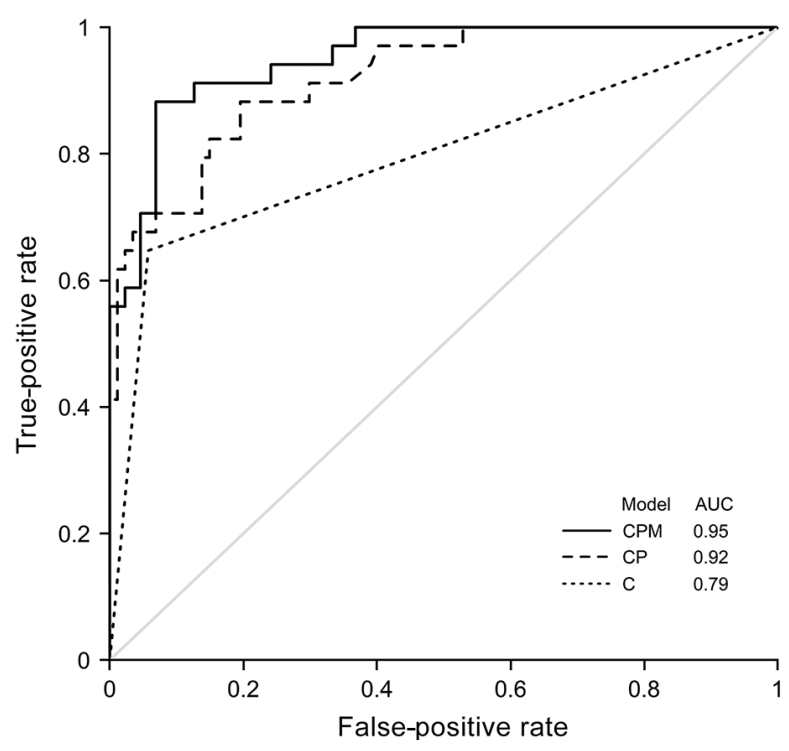

Figure 2. Receiver operating characteristics for different models in study of clinical assessments to distinguish Zika and dengue virus infections, Singapore. AUC is shown for different models: conjunctivitis alone (model $\mathrm{C}$ ), conjunctivitis with platelet count (model CP), and conjunctivitis with platelet and monocyte counts (model CPM). AUC, area under the curve. 
Table 1. Patient profile for validation cohort in study of clinical assessments to distinguish Zika and dengue virus infections, Singapore

\begin{tabular}{lc}
\hline Characteristic & Value, $\mathrm{N}=95$ \\
\hline Age & Median 38, mean 37.9, range 21-67 \\
Sex & M 72, F 23 \\
Day of illness* & Median 5, mean 4.7, range 2-9 \\
Conjunctivitis & Yes 13, no 82 \\
Fever & Yes 93, no 2 \\
Myalgia & Yes 33, no 62 \\
Headache & Yes 33, no 62 \\
Monocyte count, $\times 10^{9} / \mu \mathrm{L}$ & Median 0.32, mean 0.39, range 0.08-1.38 \\
Platelet count, $\times 10^{9} / \mathrm{LL}$ & Median 99, mean 115.2, range 13-308 \\
Alanine aminotransferase, $\mathrm{U} / \mathrm{L}$ & Median 33, mean 55.2, range 12-677 \\
Aspartate aminotransferase, $\mathrm{U} / \mathrm{L}$ & Median 44, mean 76.5, range 17-715 \\
\hline${ }^{*}$ Day on which care was sought. & \\
\hline
\end{tabular}

1), resulting in an AUC of 0.90. Applying a cutoff score of 0.34 as determined by Youden's index to maximize sensitivity and specificity of our original model to the new validation dataset, the positive predictive value was $83 \%$ and negative predictive value $87 \%$, achieving a similar diagnostic accuracy of $86 \%$.

Zika virus and DENV coexist in many developing nations in equatorial South America and Southeast Asia, where there is limited accessibility to health resources and virus-specific diagnostics are not readily available. Differentiating Zika virus and DENV infections early is important in the prognostication and subsequent monitoring and follow-up of these patients. Although Zika virus infection is self-limiting, concerns about its sequelae in pregnant women and birth defects are well established (6). In contrast, severe DENV infection leads to debilitating illness that can cause vascular leakage, dengue shock, and death (7).

We applied both definitions from the US Centers for Disease Control and Prevention (CDC) and World Health Organization (WHO) for suspected Zika cases $(8,9)$ in our patient cohort and found them to be unsatisfactory in distinguishing Zika virus from DENV patients (CDC, sensitivity $100 \%$, specificity $2 \%$; WHO, sensitivity $71 \%$, specificity $67 \%$ ) (Table 2). We therefore sought to develop more accurate indices to identify Zika virus among the backdrop of DENV cases in Singapore.

Our results highlight the utility of conjunctivitis and normal platelet and monocyte counts to distinguish Zika virus infection. We found conjunctivitis to be already a strong predictor of Zika virus infection. The study by Waggoner et al. in Nicaragua had reported conjunctivitis and rash in association with Zika virus infection (10). However, rash was not prominent among Zika patients in our study. Headache and myalgia were more common in DENV (7) and could help to distinguish DENV from Zika virus in our cohort. Prior studies had not ascertained if incorporation of basic laboratory indices could further enhance diagnostic capability. In our univariate logistic regression model, thrombocytopenia, transaminitis, and monocytosis were notable in DENV infection. Conversely, Zika patients tended to have normal platelet, aminotransaminase, and monocyte levels.

\section{Conclusions}

We were able to derive 3 simple clinical predictors on the basis of our findings: in the presence of conjunctivitis and normal platelet and monocyte counts, diagnostic AUC for Zika increased from 0.79 to 0.95 , with $92 \%$ accuracy ( $88 \%$ sensitivity and $93 \%$ specificity). The accuracy of our derived indices exceeds that of WHO's and CDC's definitions for Zika case identification, notwithstanding that performance may differ with disease prevalence or population factors. Distinguishing Zika virus from DENV infection on clinical grounds remains daunting, and it will be ideal to validate these derived indices in a prospective patient cohort. Until then, these simple clinical assessments using conjunctivitis and basic blood count parameters will be helpful in regions of the world where both Zika virus and DENV are endemic.

\begin{tabular}{|c|c|c|c|c|c|c|c|}
\hline \multicolumn{2}{|r|}{ Case definition } & \multicolumn{6}{|c|}{ Characteristic } \\
\hline Source & Criteria & $\begin{array}{c}\text { Patient meets } \\
\text { criteria }\end{array}$ & $\begin{array}{c}\text { Zika virus } \\
\text { positive, } n=34\end{array}$ & $\begin{array}{c}\text { Zika virus } \\
\text { negative, } n=57\end{array}$ & $\begin{array}{l}\text { Total, } \\
\mathrm{n}=91\end{array}$ & $\begin{array}{c}\text { Sensitivity, } \\
\%\end{array}$ & $\begin{array}{c}\text { Specificity, } \\
\%\end{array}$ \\
\hline CDC & $\begin{array}{l}\text { Clinically compatible illness with } \\
\geq 1 \text { of the following not explained } \\
\text { by another etiology: fever, rash, } \\
\text { arthralgia, or conjunctivitis } †\end{array}$ & Yes & 34 & 56 & 90 & 100 & 2 \\
\hline $\mathrm{WHO}$ & $\begin{array}{l}\text { Fever and/or rash and any of } \\
\text { the following: arthralgia, arthritis, } \\
\text { nonpurulent conjunctivitis }\end{array}$ & $\begin{array}{l}\text { Yes } \\
\text { No }\end{array}$ & $\begin{array}{l}24 \\
10\end{array}$ & $\begin{array}{l}19 \\
38\end{array}$ & $\begin{array}{l}43 \\
48\end{array}$ & 71 & 67 \\
\hline
\end{tabular}


L.Y.A.C. has been supported by the Clinician Scientist Award (CSA), Individual Research Grant (IRG), Bedside \& Bench (B\&B) grants, Centre Grant, and the Training Fellowship Award from the National Medical Research Council (NMRC),

Singapore. He also receives funding from the Aspiration Grant \& Summit Research Program and Bench to Bedside Grant from the National University Health System, Singapore, as well as the Synthetic Biology Research \& Development Program of the National Research Foundation, Singapore.

\section{About the Author}

Dr. Yan is currently an infectious diseases senior resident in the Department of Medicine at the National University Hospital of Singapore. His research interest is in rapid pathogen diagnostics.

\section{References}

1. Ooi EE, Goh KT, Gubler DJ. Dengue prevention and 35 years of vector control in Singapore. Emerg Infect Dis. 2006;12:887-93. http://dx.doi.org/10.3201/eid1206.051210

2. Musso D, Gubler DJ. Zika virus. Clin Microbiol Rev. 2016;29:487524. http://dx.doi.org/10.1128/CMR.00072-15

3. Fisher D, Cutter J. The inevitable colonisation of Singapore by Zika virus. BMC Med. 2016;14:188. http://dx.doi.org/10.1186/ s12916-016-0737-9

4. Lanciotti RS, Kosoy OL, Laven JJ, Velez JO, Lambert AJ, Johnson AJ, et al. Genetic and serologic properties of Zika virus associated with an epidemic, Yap State, Micronesia, 2007. Emerg Infect Dis. 2008;14:1232-9. http://dx.doi.org/10.3201/ eid1408.080287

5. Ho HJ, Wong JGX, Mar Kyaw W, Lye DC, Leo YS, Chow A. Diagnostic accuracy of parameters for Zika and dengue virus infections, Singapore. Emerg Infect Dis. 2017;23:2085-8. http://dx.doi.org/10.3201/eid2312.171224

6. Schuler-Faccini L, Ribeiro EM, Feitosa IM, Horovitz DD, Cavalcanti DP, Pessoa A, et al.; Brazilian Medical Genetics Society-Zika Embryopathy Task Force. Possible association between Zika virus infection and microcephaly_Brazil, 2015. MMWR Morb Mortal Wkly Rep. 2016;65:59-62. http://dx.doi.org/ 10.15585/mmwr.mm6503e2

7. Simmons CP, Farrar JJ, Nguyen vVC, Wills B. Dengue. N Engl J Med. 2012;366:1423-32. http://dx.doi.org/10.1056/ NEJMra1110265

8. Pan American Health Organization/World Health Organization. Zika resources: case definitions. [cited 2017 Oct 17]. http://www.paho.org/hq/index.php?option=com_content\&view=arti cle\&id $=11117 \&$ Itemid $=41532$

9. Centers for Disease Control and Prevention; National Notifiable Diseases Surveillance System. Zika virus disease and Zika virus infection 2016 case definition, Approved June 2016 [cited 2017 Oct 17]. https://wwwn.cdc.gov/nndss/conditions/zika/casedefinition/2016/06/

10. Waggoner JJ, Gresh L, Vargas MJ, Ballesteros G, Tellez Y, Soda KJ, et al. Viremia and clinical presentation in Nicaraguan patients infected with Zika virus, chikungunya virus, and dengue virus. Clin Infect Dis. 2016;63:1584-90. http://dx.doi.org/10.1093/cid/ciw589

Address for correspondence: Louis Yi Ann Chai, National University Health System - Division of Infectious Diseases, University Medicine Cluster, NUHS Tower Block, 1E Kent Ridge Rd, Singapore 119228, Singapore; email: chailouis@hotmail.com

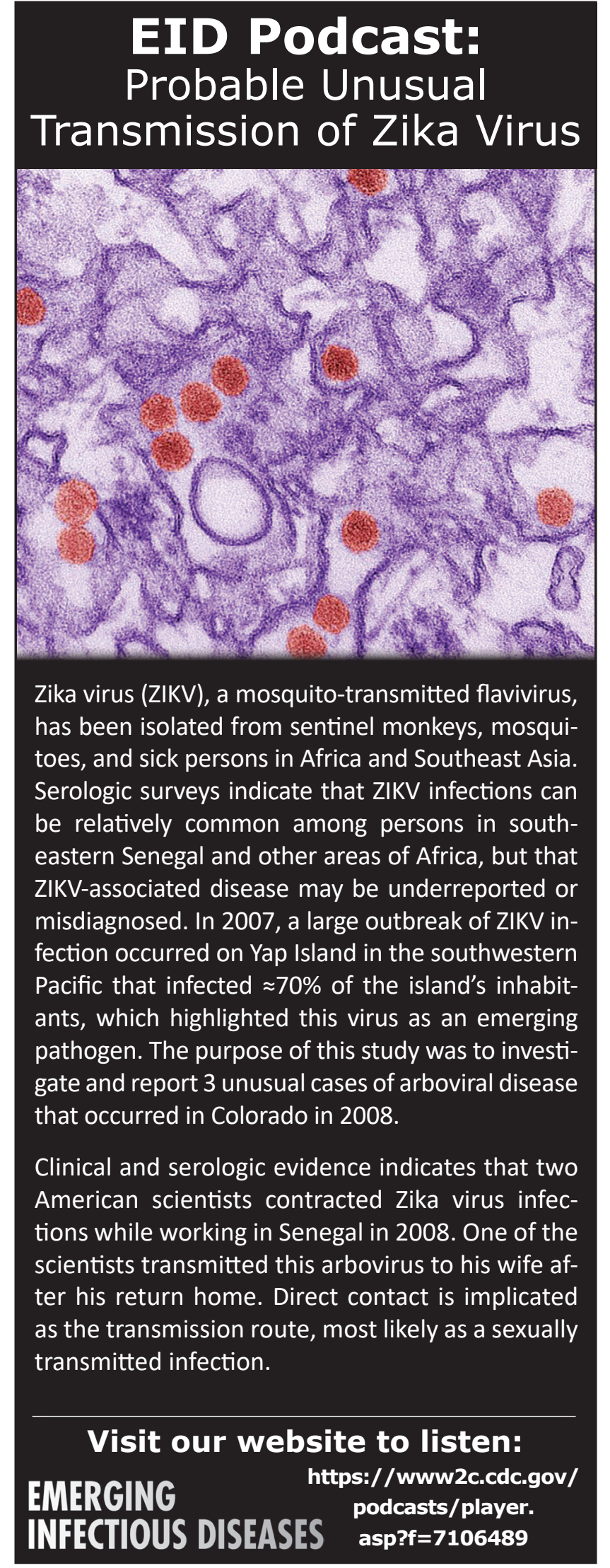

